\title{
ON THE ALGEBRAIC STRUCTURE OF DISCONTINUOUS GROUPS
}

\author{
MURRAY GERSTENHABER ${ }^{1}$
}

The purpose of this paper is to determine what may be said, under favorable conditions, about the algebraic structure of a group of homeomorphisms when a fundamental domain is known.

If $S$ is a space and $G$ a group of homeomorphisms acting on $S$, then the image of a point $x$ in $S$ under an element $g$ in $G$ will be denoted by $g x$. If $D$ is a subset of $S$, then $g D$ will be the image of $D$, and if $F$ is a subset of $G$, then $F x$ will denote the union of the images $g x$ of $x$ and $F D$ will denote the union of the sets $g D$ for all $g$ in $F$. The empty set will be denoted by $\varnothing$, the identity element of a group by 1 , and intersections, inclusions, and closures will be indicated in the usual ways.

Let there be given a connected and locally connected Hausdorff space $S$, a group $G$ of homeomorphisms acting on $S$, and a connected subspace $D$ of $S$ satisfying the following conditions:

1. $S$ is the disjoint union of the sets $g D$ with $g \in G$.

2. Let $F=\{g \in G \mid g \bar{D} \cap \bar{D} \neq \varnothing\}$. Then the number of elements in $F$ is finite and $F D$ contains a neighborhood of $D$.

If the foregoing conditions are satisfied, then in order that these concepts have names, $S$ will be called an admissible space, $G$ a group of the first kind acting on $S$, and $D$ a proper fundamental domain for $G$. It can be verified without great difficulty that the elements of $F$ generate $G,{ }^{2}$ and $F$ will be called the local set of generators relative to $D$. $D$ will be fixed throughout, and the phrase "relative to $D$ " will generally be omitted. It is evident that $1 \in F$ and that $g \in F$ implies $g^{-1} \in F$. If the space $S$ is simply connected, then the relations which hold between the local generators can be found. To state the main theorem.precisely it is necessary to introduce certain groups of which $G$ is a homomorphic image.

Let $\tilde{H}$ be an abstract free group the generators of which are the elements of $F$ different from the identity. To avoid confusion it will be assumed that a mapping $\sigma$ is given which takes the elements of $F$ different from the identity, considered as a subset of $G$, onto the generators of $\tilde{H}$, and that $\sigma$ is extended to all of $F$ by setting $\sigma(1)=1$. In $\tilde{H}$ form the smallest normal subgroup $R$ containing all the elements

Received by the editors January 17, 1953.

1 Frank B. Jewett Fellow.

The demonstration is omitted because it is already present in the literature; cf. C. L. Siegel [1]. 
$\sigma\left(g_{1}\right) \sigma\left(g_{2}\right) \sigma\left(g_{1} g_{2}\right)^{-1}$ with $g_{1}, g_{2}$, and $g_{1} g_{2}$ all in $F$, and let $H$ denote the quotient group of $\tilde{H}$ modulo $R$. The mapping $\sigma$ of $F$ into $\tilde{H}$ followed by the homomorphism of $\tilde{H}$ onto $H$ produces a mapping of $F$ into $H$ which is again one-to-one. Since $\widetilde{H}$ will be little used in the remainder of this paper, the latter mapping will also be denoted by. $\sigma$. Then in $H$ it is the case that $\sigma\left(g_{1}\right) \sigma\left(g_{2}\right)=\sigma\left(g_{1} g_{2}\right)$ whenever $g_{1}, g_{2}$, and $g_{1} g_{2}$ are in $F$. Letting $g_{2}=g_{1}^{-1}$, it follows that $\sigma\left(g^{-1}\right)=\sigma(g)^{-1}$.

There is a natural homomorphism of $\tilde{H}$ onto $G$ defined by taking the elements of $F$ considered as generators of $\tilde{H}$ back to the same elements considered as elements of $G$. The elements of the kernel of this homomorphism will be called the relations of $G$. The kernel contains $R$ and the homomorphism in question induces a natural homomorphism of $H$ onto $G$, which will be denoted by $\phi$. If $\sigma$ is considered as a mapping of $F$ into $H$ (it will always be so considered henceforth), then $\sigma$ followed by $\phi$ is the identity mapping on $F$. The elements of the group $R$ will be called the local relations. $H$ itself will be called the local universal covering group of $G$ and $\phi$ will be called the local covering homomorphism. This terminology may be partly justified by the main theorem which can now be stated.

Theorem. Let there be given an admissible space $S$, group of the first kind $G$ acting on $S$, and proper fundamental domain $D$ for $G$, and let $H$ be the local universal covering group of $G$ and $\phi$ the local covering homomorphism. Then there exists an admissible (hence in particular, connected) space $T$ with the properties:

1. $T$ is a covering space of $S$. The covering mapping will be denoted by $\psi$

2. $H$ (or more properly, a group isomorphic to $H$ ) acts as a group of homeomorphisms of the first kind on $T$ and has a proper fundamental domain $E$ homeomorphic to $D$; in fact $\psi$ is a homeomorphism on $a$ neighborhood of $E$ and $\psi(E)=D$.

3. If $y$ is a point of $T$ and $h$ an element of $H$, then $\psi(h y)=\phi(h) \psi(y)$.

There is commutativity in the diagram:

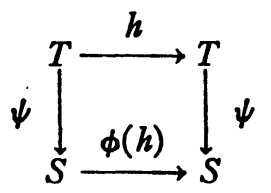

The following corollary is an immediate consequence.

CoROLlary. If $S$ is simply connected, then $G$ is isomorphic to $H$; all the relations of $G$ are local. 
Proof of THE MAIN THEOREM. (The proof will contain five lemmas.) The set $F$ of local generators being as before, let $F D$ (which may be called the star of $D$ ) be denoted by $D^{*}$. For every element $h$ of $H$ take a copy of the fundamental domain $D$ and let this copy be denoted by $h E$. The copy corresponding to the identity of $H$ may be denoted simply by $E$. Let the union of the sets $h E$ be denoted by $T . T$, when suitably topologized, will be the space desired.

If $x$ is a point of $D$, let $h x$ denote the corresponding point in the copy $h E$ of $D$. $H$ acts as a group of one-to-one transformations on $T$ by left multiplication. The homomorphism $\phi$ induces a mapping $\psi$ of $T$ onto $S$ defined by $\psi(h x)=\phi(h) x$. Conclusion 3 of the main theorem follows automatically. $T$ will be topologized so that $\psi$ is a covering mapping.

The set $\sigma(F) E$ will be denoted by $E^{*}$. Since $\sigma$ followed by $\phi$ is the identity on $F$, it follows that $\psi$ is one-to-one on any set of the form $h E^{*}$. Define a subset of $h E^{*}$ to be open if its image under $\psi$ is open and let these open sets form a subbase for the open sets of $T ; \psi$ is then continuous and $H$ a group of the first kind with proper fundamental domain $E$. Since $D^{*}$ was assumed to contain a neighborhood of $D$, this does define a topology in $T$. From the definition of $\psi$, however, it is evident only that $\psi$ is continuous but not that it is open. The next few lemmas are preparatory to the proof of that fact.

LEMMA 1. If $h_{1}$ and $h_{2}$ are in $H$, then $\psi\left(h_{1} E^{*} \cap h_{2} E^{*}\right)$ is relatively open in $\psi\left(h_{1} E^{*}\right) \cap \psi\left(h_{2} E^{*}\right)$.

Proof. Let $\psi\left(h_{1} E^{*}\right) \cap \psi\left(h_{2} E^{*}\right)$ be denoted by $X$ and $\psi\left(h_{1} E^{*} \cap h_{2} E^{*}\right)$ be denoted by $Y$. It will be proved by contradiction that $X-Y$ is relatively closed in $X$.

Suppose $x$ is in the closure in $X$ of $X-Y$ but not in $X-Y$. Now, $X, Y$, and $X-Y$ are contained in $\phi\left(h_{1}\right) D^{*}$ and are each disjoint unions of a finite number of images $g D$ of $D$ under elements $g$ of $F$. Since $x$ is in the closure of $X-Y, x$ must already be in the closure of some $g D$ contained in $X-Y$ because of the finiteness of the number of these. Since this $g D$ is contained in $X$ it must be a set of the form $\phi\left(h_{1}\right) g_{1} D$ which is also of the form $\phi\left(h_{2}\right) g_{2} D$, where $g_{1}$ and $g_{2}$ are in $F$. Therefore (1): $\phi\left(h_{1}\right) g_{1}=\phi\left(h_{2}\right) g_{2}$ since $D$ is a fundamental domain for $G$. It must be that (2): $h_{1} \sigma\left(g_{1}\right) \neq h_{2} \sigma\left(g_{2}\right)$ or $g D$ would.be in $Y$ contrary to assumption. Note again that $x$ is in $\phi\left(h_{1}\right) g_{1} \bar{D}$.

But $x$ was assumed to be not in $X-Y$, which means that $x$ is in $Y$. Being in $X$, it is therefore in a set of the form $\phi\left(h_{1}\right) g_{8} D$ which is also of the form $\phi\left(h_{2}\right) g_{4} D$ implying as before that $(3): \phi\left(h_{1}\right) g_{3}=\phi\left(h_{2}\right) g_{4}$, where in addition, (4): $h_{1} \sigma\left(g_{3}\right)=h_{2} \sigma\left(g_{4}\right)$ in order that $x$ may be in $Y$. 
Since $x$ was in both $\phi\left(h_{1}\right) g_{1} \bar{D}$ and $\phi\left(h_{1}\right) g_{3} D$, it follows that $\phi\left(h_{1}\right) g_{1} \bar{D}$ $\cap \phi\left(h_{1}\right) g_{3} D \neq \varnothing$, whence $g_{1} \bar{D} \cap g_{3} D \neq \varnothing$. Therefore $g_{1}^{-1} g_{3}$ is in $F$. But from (1) and (3) we have $g_{1}^{-1} g_{3}=g_{2}^{-1} g_{4}$. Therefore (5): $\sigma\left(g_{1}\right)^{-1} \sigma\left(g_{3}\right)$ $=\sigma\left(g_{1}^{-1} g_{3}\right)=\sigma\left(g_{2}^{-1} g_{4}\right)=\sigma\left(g_{2}\right)^{-1} \sigma\left(g_{4}\right)$, by the manner in which $H$ was constructed. Combining (5) with (2) one obtains that $h_{1} \sigma\left(g_{8}\right)$ $\neq h_{2} \sigma\left(g_{4}\right)$, which contradicts (4). This proves the lemma.

LEMma 2. Let there be given sets $T, S$, a mapping $\psi: T \rightarrow S$, and subsets $N_{1}, O_{1}, N_{2}, O_{2}$ of $T$ such that $N_{1} \supset O_{1}$ and $N_{2} \supset O_{2}$. Suppose that the restriction of $\psi$ to $N_{1}$ is one-to-one and that the restriction of $\psi$ to $N_{2}$ is likewise one-to-one. Then $\psi\left(O_{1} \cap O_{2}\right)=\psi\left(O_{1}\right) \cap \psi\left(O_{2}\right) \cap \psi\left(N_{1} \cap N_{2}\right)$.

The proof of this lemma is not difficult and is omitted. Lemma 2 was phrased for arbitrary sets and a mapping between them; now let $T, S$, and $\psi$ be as before.

Lemma 3. $\psi: T \rightarrow S$ is an open mapping.

Proof. On sets of the form $h E^{*}, \psi$ is one-to-one, and a subbase for the open sets of $T$ was obtained by letting a subset of $h E^{*}$ be open if its image under $\psi$ was open. Therefore $\psi$ maps an open set of the given subbase onto an open set. It must be shown that $\psi$ does the same to intersections of subbasic open sets. For this it is sufficient to prove the following: If $O_{1}$ is an open set of $T$ contained in a set of the form $h_{1} E^{*}$ and $O_{2}$ an open set of $T$ contained in a set of the form $h_{2} E^{*}$, and if $\psi\left(O_{1}\right)$ and $\psi\left(O_{2}\right)$ are both open, then $\psi\left(O_{1} \cap O_{2}\right)$ is also open. Since $\psi$ is one-to-one on sets of the form $h E^{*}$, Lemma 2 may be applied and one obtains $\psi\left(O_{1} \cap O_{2}\right)=\psi\left(O_{1}\right) \cap \psi\left(O_{2}\right) \cap \psi\left(h_{1} E^{*} \cap h_{2} E^{*}\right)$. Since $\psi\left(O_{1}\right)$ and $\psi\left(O_{2}\right)$ are open, it follows from Lemma 1 that this is relatively open in $\psi\left(O_{1}\right) \cap \psi\left(O_{2}\right) \cap \psi\left(h_{1} E^{*}\right) \cap \psi\left(h_{2} E^{*}\right)$. But this is just $\psi\left(O_{1}\right)$ $\cap \psi\left(O_{2}\right)$, since $O_{1} \subset h_{1} E^{*}$ and $O_{2} \subset h_{2} E^{*}$. Therefore $\psi\left(O_{1} \cap O_{2}\right)$ is relatively open in $\psi\left(O_{1}\right) \cap \psi\left(O_{2}\right)$. But the latter is an open set, so it follows that the former is also, which proves the lemma.

From the fact that $\psi$ is continuous, open, and one-to-one on sets of the form $h E^{*}$ it follows that $\psi$ is a homeomorphism on such sets; conclusion 2 of the main theorem follows from this.

Nothing so far proved implies that $T$ is a Hausdorff space. This could be shown directly, but will also follow automatically from the fact that $T$ covers $S$.

LeMma 4. $\psi: T \rightarrow S$ is a covering mapping.

Proof. Let a point $x$ in $S$ be given; we may assume it is in $D$. It must be shown that there exists a neighborhood $U$ of $x$ such that the inverse image under $\psi$ of $U$ is a disjoint union of open sets each of 
which is mapped homeomorphically onto $U$ by $\psi$. But there exists a neighborhood $U$ of $x$ which is contained in $D^{*}$, since it was assumed that $D^{*}$ contains in fact a neighborhood of $D$. It will be shown that such a $U$ will serve. Let $K$ denote the kernel of $\phi ; K$ will be the group of covering homeomorphisms.

For every $h_{i}$ in $K$, form the set $\psi^{-1}(U) \cap h_{i} E^{*}$, which will be denoted by $U_{i} . U_{i}$ is open since it is contained in a set of the form $h E^{*}$ and its image under $\psi$, which is $U$, is open; all such sets were in the subbasis for the open sets of $T$ by means of which the topology of $T$ was defined. Furthermore $\psi$ maps $U_{i}$ homeomorphically on $U$. The inverse image of $U$ under $\psi$ is the union of the sets $U_{i}$ and it is therefore sufficient to prove that the sets $U_{i}$ are disjoint. Suppose not: say $U_{1} \cap U_{2}$ $\neq \varnothing$. $U_{1}$ and $U_{2}$ each contain exactly one inverse image of $x$. Let these points be called $y_{1}$ and $y_{2}$ respectively, and let $z$ be a point in $U_{1} \cap U_{2}$. Then $z$ is in a set of the form $h_{1} \sigma\left(g_{1}\right) E$ which is also of the form $h_{2} \sigma\left(g_{2}\right) E$, where $g_{1}$ and $g_{2}$ are in $F$. This implies that $h_{1} E$ and $h_{2} E$ are both in $h_{1} \sigma\left(g_{1}\right) E^{*}=h_{2} \sigma\left(g_{2}\right) E^{*}$. But $\psi$ can not then be a homeomorphism on $h_{1} \sigma\left(g_{1}\right) E^{*}$, for this set contains both $y_{1}$ and $y_{2}$, which have the same image. This contradiction shows that the sets $U_{i}$ are indeed disjoint and ends the proof of the lemma. The assumption that $S$ is locally connected has tacitly entered here; it is otherwise not even meaningful to speak of a covering space of $S$.

Lemma 4 proves conclusion 1 of the main theorem. It remains only to prove that $T$ is an admissible space. Since $\psi$ is a local homeomorphism, it is evident that $T$ will be localled connected. All that remains, then, is to show that $T$ is connected.

LEMMA 5. $T$ is connected.

Proof. $D$ is connected and $\psi$ is a homeomorphism on sets of the form $h E^{*}$ with $h$ in $H$. Therefore $E$ and every set of the form $h E$ with $h$ in $H$ is connected. If $g$ is any element of $F$, then $g D$ is by assumption adherent to $D$. Therefore $\sigma(g) h E$ is adherent to $h E$ for any element $h$ of $H$, again because $\psi$ is a homeomorphism on sets of the form $h E^{*}$. Therefore, the connected component of a point of $E$ must contain all of $E$, and with any set $h E$ it must contain every set of the form $\sigma(g) h E$ with $g$ in $F$. But $\sigma(F)$ generates $H$. Therefore the connected component of any point of $E$ contains all of $T$, and so $T$ must be connected. This proves the lemma.

With the demonstration of Lemma 5, the proof of the main theorem is complete.

While the space $T$ whose existence is asserted in the main theorem is a covering space of $S$, it is in general not the universal covering but 
does have certain analogous properties. Let the triple consisting of an admissible space $S$, group $G$ of the first kind acting on $S$, and a proper fundamental domain $D$, be called a localized discontinuous group of the first kind. For simplicity, the phrase "of the first kind" will be dropped, but "localized" is included to indicate that a specific fundamental domain is given. If $\mathbb{S}=(S, G, D)$ and $\mathfrak{W}=(T, H, E)$ are two localized discontinuous groups, then $\$$ will be said to be a covering group of $B$ if

1. $T$ is a covering space of $S, G$ is a homomorphic image of $H$, and there is a covering mapping $\psi$ and a homomorphism $\phi$ satisfying 2 and 3.

2. There is a neighborhood of $E$ on which $\psi$ is a homeomorphism and $\psi(E)=D$.

3. If $y$ is a point of $T$ and $h$ an element of $H$, then $\psi(h y)=\phi(h) \psi(y)$.

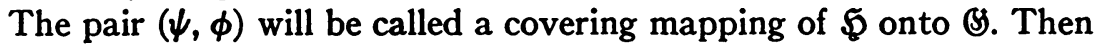
the main theorem really asserts that if a localized discontinuous group $\mathbb{B}=(S, G, D)$ is given, then there exists a covering group $\mathfrak{W}=(T, H, E)$

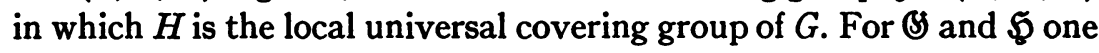
may then assert the following.

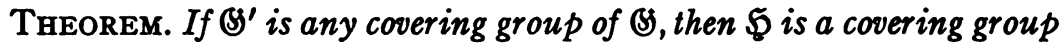
of 'B'.

The proof of this theorem is not difficult and will be omitted. It indicates the sense in which $H$ may be said to be universal and justifies calling $\mathfrak{W}$ the local universal covering group of $G$.

It is easy to give examples of the application of the main theorem. In theory, at least, it gives the structure of most of the common discontinuous groups of complex-analytic homeomorphisms operating on the upper half-plane, and in fact readily gives the structure of those groups whose fundamental domains are of a simple nature, such as the modular group.

\section{REFERENCE}

1. C. L. Siegel, Discontinuous groups, Ann. of Math. vol. 44 (1943).

The Institute for Advanced Study 